\title{
EVALUACIÓN DE LA ESTRATEGIA DE ATENCIÓN PRIMARIA CON ENFOQUE FAMILIAR "SALUD PUERTA A PUERTA" EN EL DEPARTAMENTO DEL HUILA DURANTE EL PERIODO 2006 - 2007
}

\author{
Evaluation of Strategy of Primary Attention with Family Focus "Health Door to Door" in Huila Department in 2006 - 2007.
}

Claudia Elena Ruiz Jaramillo. Especialista en Gerencia en Servicios de Salud y Seguridad Social, Facultad de Salud, Universidad Surcolombiana, Neiva.

Correspondencia: e-mail: lenaruiz2020@yahoo.es

\section{RESUMEN}

En el Departamento del Huila, se implementó la estrategia de Atención Primaria en Salud (APS), con Enfoque Familiar "Salud Puerta a Puerta" (EF SPP), con el propósito de contribuir a la disminución de los indicadores de morbimortalidad, en la población rural pobre y urbana marginal, con difícil acceso a los servicios de salud y con múltiples factores de riesgo.

Después de casi tres años de establecimiento de la estrategia, es indispensable un proceso de evaluación de los resultados, que proporcione herramientas de apoyo, en las acciones encaminadas a establecer métodos eficaces para el fortalecimiento de la APS. Para ello se consolidólainvestigación, medianteun marcoconceptual mínimo general y una revisión completa y crítica de los resultados de APS SPP para reflejar las necesidades comunes de salud y desarrollo de la población huilense. La evaluación arroja un porcentaje promedio de $88 \%$ lo que corresponde a una calificación de ACEPTABLE, demostrando grandes debilidades en variables como la Promoción y la Prevención y en los mecanismos de participación activa, elementos relevantes de la Atención Primaria en Salud, igualmente, se presentan en condiciones aptas las variables de aceptabilidad, recursos humanos apropiados y la intersectorialidad. Las variables calificadas como optimo son accesibilidad, demanda inducida, integralidad, primer contacto, enfoque poblacional atención apropiada e integración FESP, todas excepto accesibilidad y primer contacto fueron evaluadas cualitativamente ya que para el proceso de investigación es inaplicable determinar un valor cuantitativo, y no existen documentalmente los valores correspondientes.

\section{PALABRAS CLAVE}

Sistemas de cuidado de salud, Atención Primaria en Salud.

\section{ABSTRACT}

In the Department of Huila, it was implemented the Primary Health Care (PHC), strategy with a "Door to Door Health" family approach. The strategy aims to contribute to the decrease in morbidity and mortality rates in the marginal poor, urban and rural population with difficult access to the care services of health and with multiple risk factors.

After almost three years of having been implemented, it is indispensable a process of evaluation of the results. It will provide supporting tools for the steps taken towards the development of effective methods to strengthen the PHS strategy. For that purpose the research was consolidated through the drawing up of a general basic conceptual framework and a complete and critical revision of the results of PHC - DDH in order to know the common health and development needs of Huila population. The evaluation shows an average percentage of $88 \%$ which means ACCEPTABLE, with big weaknesses in variables like promotion and prevention and in the active participation mechanisms which are relevant elements of the Primary Health Care. Likewise, variables such as acceptability, appropriate human resources, and the intersectorial aspect are considered to be under acceptable conditions. 
The variables considered as optimum are accessibility, induced demands, integrality, first contact, population approach, appropriate service and FESP integration. All but accessibility and first contact were evaluated qualitatively since for the investigation process it is inapplicable to determine a quantitative value, and the corresponding values don't appear in the documents.

\section{KEY WORDS}

Delivery of Health Care, Health Care Systems

\section{INTRODUCCIÓN}

La organización de los componentes de un sistema de salud, basado en la Atención Primaria en Salud, está organizado en valores, principios y elementos. Los valores son esenciales para establecer las prioridades nacionales y para evaluar si los arreglos sociales, responden o no a las necesidades y expectativas de la población ${ }^{1}$; El derecho a la salud, La equidad en salud y La solidaridad; Los principios, otorgan los cimientos para las políticas de salud, la legislación, los criterios evaluativos, la generación y asignación de los recursos, y para la operación del sistema de salud $^{2}$. Responder a las necesidades de salud, La responsabilidad y rendición de cuentas, Los servicios orientados hacia la calidad, La justicia social, La sostenibilidad, La intersectorialidad, Participación individual y social; y los elementos: componentes organizacionales y funcionales, que permiten organizar las políticas, los programas y los servicios, que deben estar interconectados y fundamentados en evidencias que prueben su efectividad, para mejorar la salud3. La accesibilidad, La aceptabilidad, Primer contacto, La integralidad, Atención integrada El enfoque poblacional Prevención y promoción, Atención apropiada y efectiva, La efectividad, Recursos humanos apropiados, Funciones Esenciales de la Salud Pública, Acciones intersectoriales, Mecanismos de participación activa ${ }^{3}$.

\section{ESTRATEGIA DE ATENCIÓN PRIMARIA EN 'SALUD PUERTA A PUERTA'}

La Estrategia de Atención Primaria en Salud con Enfoque Familiar "Salud Puerta a Puerta" (Estrategia APSEF-SPP), fue concebida como una herramienta para contribuir al cumplimiento de los Objetivos de Desarrollo del Milenio (principalmente los objetivos
1, 4, 5 y 6). colocando a disposición de las familias los medios necesarios para manejar, controlar su salud y autocuidarse, mediante la adquisición de conocimientos y prácticas, impartidos con un enfoque en salud familiar, por un equipo básico de salud interdisciplinario y para lograr su propósito, involucra la atención integral del individuo, la familia y la comunidad, desde el punto de vista biopsicosocial, incorporando a la familia como unidad de atención y la comunidad como escenario principal de las acciones de salud. ${ }^{4}$

Dentro de los momentos importantes para la operativización de la estrategia se tiene: Primero: Conformación y organización del equipo extramural; Segundo: Aprestamiento y dotación del equipo extramural; Tercero: Acción a nivel familiar y comunitario y, Cuarto: Participación de la familia y la comunidad. ${ }^{5}$

La siguiente investigación evalúa la Estrategia de Atención Primaria con Enfoque Familiar Salud Puerta a Puerta en los períodos 2006 y 2007. Específicamente, se evalúan objetivos y metas en términos de cobertura y oportunidad, de esta estrategia además se muestra un enfoque diferente al cumplimiento contractual reflejado en los informes presentados, durante el proceso, por las partes integrantes del documento legal y busca comprender la importancia y necesidad de la investigación en la Atención Primaria

\section{MATERIALES Y MÉTODOS}

La evaluación de la APS EF SPP, se presento como un proceso de búsqueda de elementos que permitieron emitir valor cualitativo y cuantitativo sobre las actividades realizadas, específicamente de los elementos contenidos en la Renovación de la Atención Primaria.

\section{PREPARACIÓN DEL PROCESO EVALUATIVO}

Dentro de las fase de preparación del proceso tenemos: 1) Selección de las actividades evaluables "trazadores" de la efectividad de la ESPP, apoyados en: Documentos de análisis de la APS; documentos informes de Interventoría de los períodos 2006 y 2007; información derivada del Sistema de Información de APS SICAPS. La información suministrada en las bases de datos de EPIINFO, permite evaluar las actividades de detección temprana y protección 
específica, realizadas por los equipos extramurales en la población sujeto de intervención; la información de la ficha SICAPS que aporta la información para la elaboración del diagnóstico comunitario, permite la identificación de los grupos de riesgo en la población; revisión de textos (diarios, boletines, normas, declaraciones, artículos de diarios, propaganda, páginas web, presentaciones institucionales); y encuestas realizadas por la interventoría, la consolidación de información y cruce de datos para realizar la interpretación; 2) Definición de las dimensiones de la evaluación. Estimar la calidad del producto de APS, exclusivamente mediante el proceso y la estructura, mientras no sea posible valorarla en términos de resultados en salud no es viable; igualmente no todos los elementos de la APS, permiten ser evaluados con la información disponible; 3) Determinación de criterios, indicadores y estándares a utilizar en la evaluación. En esta determinación, se tuvo en cuenta los criterios de estructura, proceso y resultados, y 4) Se seleccionaron métodos evaluativos y confección de instrumentos evaluativos, Así: Observación, análisis de la información y estandarización de la información. Para la aplicación de cada método evaluativo, se confeccionaron los instrumentos correspondientes: guías, cuestionarios, tablas de vaciamiento, entre otras.

\section{EJECUCIÓN DEL PROCESO EVALUATIVO}

Para la ejecución del proceso evaluativo se tiene: 1) Aplicación de los instrumentos diseñados para evaluar los criterios seleccionados; 2) Determinación de las desviaciones existentes en relación con los estándares preestablecidos, y 3) Análisis de las desviaciones. Se aplicaron técnicas de triangulación de datos y fuentes. Para el procesamiento de datos cuantitativos, se realizaron tablas en Microsoft Excel, con base en el relevamiento documental y el análisis de fuentes secundarias.

\section{DESCRIPCIÓN DE LAS VARIABLES DE LA INVESTIGACIÓN}

La calificación, correspondió a un valor del factor de medición, determinando una apreciación máxima de 100 puntos, y cuyo valor correspondió a la equivalencia de los resultados, medidos en el proceso de interventoría. Esta puntuación tendrá una calificación de acuerdo al rango porcentual y será: Óptima: Mayor o igual 90\% del puntaje; Adecuada:
Entre $80 \%$ y $89 \%$ del puntaje y, Deficiente: Menor del $80 \%$ (Tabla 1).

Tabla 1. Descripción variable de investigación

\begin{tabular}{|c|c|}
\hline VARIABLE & INDICADOR \\
\hline ACCESIBILIDAD & $\begin{array}{l}\text { COBERTURAS VEREDAL } \\
\text { COBERTURAS FAMILIAR } \\
\text { COBERTURA POBLACIONAL }\end{array}$ \\
\hline ACEPTABILIDAD & SATISFACCIÓN DEL USUARIO \\
\hline $\begin{array}{l}\text { PRIMER } \\
\text { CONTACTO }\end{array}$ & $\begin{array}{l}\text { DEMANDA INDUCIDA } \\
\text { POR PROGRAMAS }\end{array}$ \\
\hline INTEGRALIDAD & ATENCIÓN INTEGRAL \\
\hline $\begin{array}{l}\text { ENFOQUE } \\
\text { POBLACIONAL }\end{array}$ & $\begin{array}{l}\text { INFORMACIÓN EN SALUD } \\
\text { PUBLICA } \\
\text { ENFOQUE FAMILIAR } \\
\text { ENFOQUE COMUNITARIO }\end{array}$ \\
\hline $\begin{array}{l}\text { PROMOCIÓN Y } \\
\text { PREVENCIÓN }\end{array}$ & COBERTURAS PROGRAMAS \\
\hline $\begin{array}{l}\text { ATENCIÓN } \\
\text { APROPIADA }\end{array}$ & EFECTIVIDAD \\
\hline $\begin{array}{l}\text { RECURSOS } \\
\text { HUMANOS } \\
\text { APROPIADOS }\end{array}$ & $\begin{array}{l}\text { APRESTAMIENTO } \\
\text { EVALUACIÓN EN } \\
\text { SISTEMAS DE INFORMACIÓN } \\
\text { CONFORMACIÓN }\end{array}$ \\
\hline INTEGRACIÓN FESP & $\begin{array}{l}\text { REGISTRO ESP } \\
\text { CAPACITACIÓN } \\
\text { IMPLEMENTACIÓN } \\
\text { Y OPERATIVIZACIÓN }\end{array}$ \\
\hline INTERSECTORIALIDAD & $\begin{array}{l}\text { REUNIONES DE CONCERTACIÓN } \\
\text { PLANES DE ACCIÓN FAMILIAR } \\
\text { PLANES DE ACCIÓN VEREDAL }\end{array}$ \\
\hline $\begin{array}{l}\text { MECANISMO DE } \\
\text { PARTICIPACIÓN } \\
\text { ACTIVA }\end{array}$ & $\begin{array}{l}\text { UROC Y UAIRAC } \\
\text { COVECOM } \\
\text { IIAMI Y AIEPI }\end{array}$ \\
\hline
\end{tabular}

\section{RESULTADOS Y DISCUSIÓN}

\section{LA ACCESIBILIDAD}

La selección de las zonas a intervenir, se realizó con la participación de la ESE (Empresa Social del Estado) o UAE local, y con la alcaldía municipal (Dirección Local de Salud), garantizando el cumplimiento de los criterios selectivos, que se basaron en: dificultades de accesibilidad a los servicios de salud, indicadores materno-infantil críticos, que afecten el cumplimiento de las metas, coberturas deficientes en los programas 
de Detección Temprana (DT) y Protección Especifica (PE) y Poblaciones pobres y vulnerables.

Para medir la accesibilidad, se realizó un análisis cuantitativo del cumplimiento de las coberturas, teniendo como base las actividades programadas y las ejecutadas de las coberturas de las familias, veredas y personas.
Las coberturas fueron definidas contractualmente, el resultado cuantitativo demuestra que estas mejoraron de un período a otro, en la atención de las familias y las veredas, pero se atendieron más personas en el primer periodo, debido a que en el tercer momento de intervención familiar y comunitaria, en el año 2006, fue más amplio que en el 2007 por motivos administrativos (Tabla 2).

Tabla 2. Coberturas poblacionales del programa de Atención Primaria con Enfoque Familiar

\begin{tabular}{|c|c|c|c|c|c|c|}
\hline & \multicolumn{3}{|c|}{ PERSONAS } & \multicolumn{2}{|c|}{ FAMILIAS } & \multicolumn{2}{|c|}{ VEREDAS } \\
\hline PERIODO & 2006 & 2007 & 2006 & 2007 & 2006 & 2007 \\
\hline PROGRAMADO & $136.000^{*}$ & 204.000 & 43.168 & 50.322 & 889 & 983 \\
\hline ATENDIDAS & 167.026 & 198.407 & 43.483 & 52.326 & 808 & 976 \\
\hline COBERTURA (\%) & 123 & 97 & 101 & 104 & 91 & 99 \\
\hline
\end{tabular}

*valores en miles de personas

\section{ACEPTABILIDAD}

Durante el desarrollo de la Estrategia, la interventoría realizó visitas de campo en los 35 municipios (a excepción de Saladoblanco y Gigante que no ingresaron a la estrategia en el año 2007), aplicando los formato de "ENCUESTA DE SATISFACCION DE USUARIOS APS PUERTA A PUERTA" 1 y 2 realizadas en el año 2007.

Las preguntas de la encuesta, se respondieron dando diferentes alternativas. Si, No o SR (sin respuesta); Excelente, Bueno, Regular, Malo o No Responde, variando de pregunta a pregunta. Las preguntas con la variable no aplica no se tuvieron en cuenta. Por último las respuestas registradas como Si, Excelente o Bueno, se tomaron como aceptación positiva, y las respuestas registradas como No, Regular o Malo, se tomaron de aceptación negativa.

Los resultados fueron tabulados de acuerdo al tipo de respuesta. Las preguntas contestadas como regular, no se tuvieron en cuenta por no encontrarse un patrón de relación con las respuestas de la tabla anterior. Al final, se presenta el total de los valores promedios ponderados, para la verificación de la operación (Figura 1).

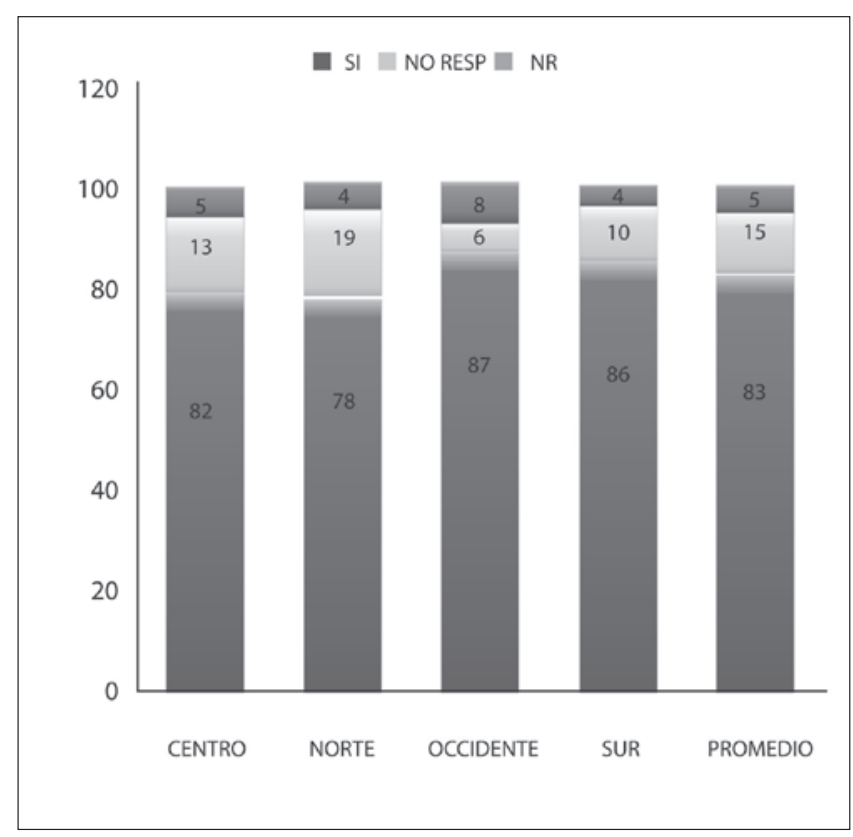

Figura 1. Promedio de las respuestas a las encuestas de satisfacción por parte de los usuarios APS SPP (USCO) 
Elgradodeaceptabilidadalresponderafirmativamente las preguntas, es de $79 \%$ en promedio. Las regiones Centro y Norte se encuentran por debajo del Promedio (Figura 2).

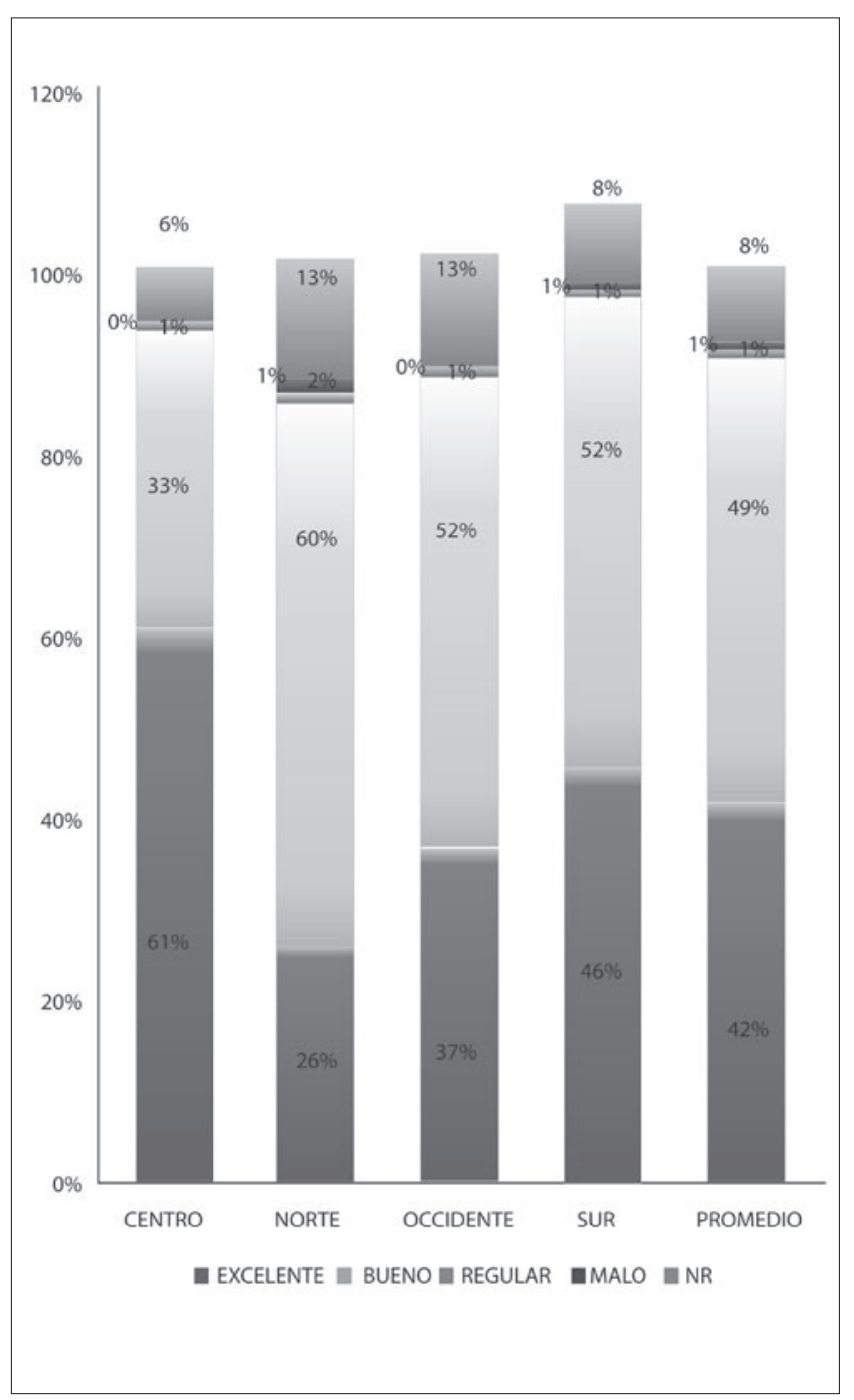

Figura 2. Porcentaje de preguntas de las encuestas de satisfacción usuarios APS SPP (USCO)

Como resultado, se promedian las respuestas de aceptabilidad, se determina un promedio final de $87 \%$ de aceptabilidad en las respuestas de la Encuesta de Satisfacción de Usuarios.

\section{PRIMER CONTACTO}

La demanda inducida, son todas las acciones encaminadas a informar y educar a la población afiliada,conelfindedarcumplimientoalasactividades, procedimientos e intervenciones de protección específica y detección temprana, establecidas en las normas técnicas. En las actividades de PE, la atención del parto y la atención del recién nacido, el Equipo Básico en Salud (EBS), realizó la demanda inducida efectiva, para que estas atenciones se realizaran de manera institucional y solamente se asistían por parte del equipo en situaciones excepcionales de urgencia (Figura 3).

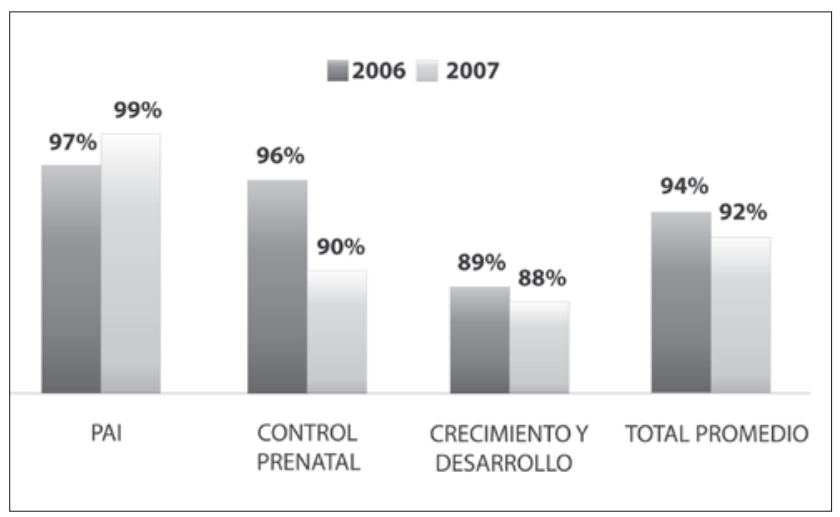

Figura 3. Porcentaje de la Demanda inducida durante el periodo de 2006-2007

En todas las actividades se realizaron controles periódicos, se presentaron los resultados de los exámenes de laboratorio y se complementó con la conducta médica, de acuerdo a los resultados; el conocimiento de los resultados de la toma de citología garantizo la continuidad de la intervención, de acuerdo al reporte, etc. El resultado del indicador, se promedia en $93 \%$ para una calificación de ÓPTIMO. Otro factor para evaluar en el elemento primer contacto, es el seguimiento a las actividades de la demanda inducida; este proceso no se llevo a cabo, durante el desarrollo de la estrategia, soportados en la falta de continuidad, limitando los momentos de intervención y consecuentemente el procedimiento de seguimiento a las actividades descritas; por lo tanto no se considero como variable a evaluar.

\section{INTEGRALIDAD}

El manejo operativo de la estrategia, involucra las actividades de referencia y contrarreferencia, lo mismo que contempla todas las actividades de los programas de protección específica y detección temprana (Resolución 412 de 2000), la vigilancia 
de eventos de interés en salud pública y atención de consulta externa prioritaria, de acuerdo a las necesidades identificadas por el EBS, a las familias pobres no aseguradas y/o nivel I y II del área rural y/o zona urbana marginal de los municipios. Los datos documentales, demuestran que se brindo atención integral, demandando información apropiada y gestión de sistemas, así como de adecuados incentivos, políticas y procedimientos, asegurando que todas las necesidades de salud fuesen satisfechas. Desafortunadamente para el proceso de investigación es inaplicable determinar un valor cuantitativo de la integralidad, ya que no existen documentalmente valores correspondientes a los estados de referencia y contrarreferencia. Sin embargo, para determinar el indicador, se tuvo en cuenta más en sentido cualitativo en cuanto a la aplicación de su estructura metodológica, que comprendió los principios de integralidad, dándose un valor de $100 \%$.

\section{ENFOQUE POBLACIONAL}

La Estrategia APSEF-SPP, tiene como característica fundamental, el enfoque de salud familiar, donde la familia y su entorno se constituyen en la Unidad Básica de las acciones integrales, para el mantenimiento y mejoramiento de su estado de salud. El diligenciamiento de la tarjeta SICAPS, se constituye en una herramienta fundamental, que facilita el análisis de la información, el ordenamiento de los datos, obtención de indicadores predeterminados para la realización del diagnóstico comunitario, la elaboración de planes de acción familiar y veredal, la planeación y programación de actividades de protección específica y detección temprana. Por último las actividades colectivas, constituyen el componente de educación en salud dirigido a la comunidad, que pretende facilitar a las personas la búsqueda de alternativas de un mejor estar de vida en un continuo desarrollo de la comunidad. Sin embargo, el parámetro para adoptar el valor del indicador, no permite dar precisión cuantitativa de cada unidad a evaluar; se estimó la observancia de los componentes del enfoque poblacional, para dar el porcentaje de cumplimiento.

El promedio de todas las actividades de protección específica y detección temprana, es de $62 \%$. Para medir el grado de cobertura de las actividades esenciales cuyo principio fundamental es la disminución de la morbi-mortalidad en las mujeres embarazadas y niños menores de un año (Tabla 3).
Tabla 3. Promedio actividades de Detección Temprana y Protección Específica de enfermedades

\begin{tabular}{|c|c|c|c|c|}
\hline PROGRAMA & PROCEDIMIENTO & \begin{tabular}{c|c|}
$\%$ \\
2006 \\
\end{tabular} & \begin{tabular}{c|c|}
$\%$ \\
2007 \\
\end{tabular} & $\begin{array}{c}\% \\
\text { PROMEDIO }\end{array}$ \\
\hline \multirow{2}{*}{ PAI } & CONTRA (DPT) & $60^{*}$ & 52 & 56 \\
\hline & $\begin{array}{c}\text { CONTRA } \\
\text { TRIPLE VIRAL }\end{array}$ & 86 & 79 & 83 \\
\hline CPF & $\begin{array}{l}\text { CONSULTA } \\
1 \text { A VEZ MG }\end{array}$ & 57 & 54 & 56 \\
\hline CYD & $\begin{array}{l}\text { CONSULTA } \\
\text { MEDICA }\end{array}$ & 89 & 83 & 86 \\
\hline JOVEN & $\begin{array}{l}\text { CONSULTA } \\
\text { 1A VEZ MG }\end{array}$ & 70 & 59 & 65 \\
\hline CPN & $\begin{array}{l}\text { CONSULTA } \\
\text { 1A VEZ MG }\end{array}$ & 99 & 97 & 98 \\
\hline CCU & $\begin{array}{c}\text { CITOLOGÍA } \\
\text { CERVICOUTERINA }\end{array}$ & 61 & 66 & 64 \\
\hline
\end{tabular}

El promedio de las actividades prioritarias es de $73 \%$. Las actividades de planificación familiar en los rangos de 10 a 14 años no se tuvieron en cuenta, por inconvenientes de tipo cultural que no permitieron un desarrollo normal. En el programa de detección temprana de alteraciones del embarazo, se encuentra una cobertura del $90 \%$, igual que la meta establecida, las alteraciones del crecimiento y desarrollo en menores de 10 años, se encuentra una cobertura del $86 \%$ inferior a la de la meta establecida, que es del 90\%. Para el cumplimiento de algunas coberturas, se presentaron situaciones ajenas al compromiso y la voluntad de los EBS, que impidieron la ejecución de algunas actividades programadas; como falta de personal médico y enfermeras, municipios con fuentes hídricas con alto contenido de flúor, menores con índices de fluorosis, dificultad para realizar actividades por concentración, por la labor agrícola y del hogar del habitante rural, condiciones de tipo cultural y religioso para los dos rangos, poca colaboración desde los encargados del PAI en las ESE-UAE del primer nivel. En algunos municipios no se les permitió aplicar los temas biológicos; deficiencia de reporte por los municipios y regionales en la atención del parto, constante movilización de la población joven de la zona rural (desplazamientos entre veredas o fincas por la época de cosecha), entre otras (Tabla 4). 
Tabla 4. Cobertura de Detección Temprana y Protección Específica de enfermedades.

\begin{tabular}{|c|c|c|c|c|}
\hline $\begin{array}{l}\text { ACTIVIDADES } \\
\text { DE PE Y DT }\end{array}$ & FACTOR DE COBERTURA & $\begin{array}{c}\% \\
2006\end{array}$ & $\begin{array}{c}\% \\
2007\end{array}$ & PROMEDIO \\
\hline \multirow{8}{*}{ PAl } & BCG $<1$ & $97^{*}$ & $92^{*}$ & $95^{*}$ \\
\hline & ANTI $\mathrm{HB}<1$ & 60 & 48 & 54 \\
\hline & DPT $<1$ & 60 & 52 & 56 \\
\hline & VOP $<1$ & 58 & 49 & 54 \\
\hline & HMOPHIL $<1$ & 60 & 51 & 56 \\
\hline & TRIPLE VIRAL & 86 & 79 & 83 \\
\hline & AMARILICA & 82 & 86 & 84 \\
\hline & TD 0 TT & 60 & 54 & 57 \\
\hline \multirow{5}{*}{ SALUD ORAL } & TOTAL SALUD ORAL & 66 & 60 & 63 \\
\hline & SELLANTES & 65 & 45 & 55 \\
\hline & DETARTRAJE & 39 & 26 & 33 \\
\hline & CONTROL DE PLACA & 59 & 43 & 51 \\
\hline & FLÚOR & 71 & 43 & 57 \\
\hline \multirow{2}{*}{ ATENCIÓN DE PARTO } & PARTOS INSTITUCIONAL & 45 & 31 & 38 \\
\hline & PARTOS DOMICILIARIOS & 4 & 6 & 3 \\
\hline ATENCIÓN DEL RN & RECIÉN NASCIDO & 25 & 19 & 22 \\
\hline CONTROL PLANIFICACIÓN & CPF & 57 & 54 & 56 \\
\hline FAMILIAR (CPF) & CPF 10 A 14 & 6 & 6 & 6 \\
\hline \multirow{4}{*}{$\begin{array}{l}\text { CONTROL Y DESARROLLO } \\
\text { ACTIVIDADES DE PE Y DT }\end{array}$} & $\%$ COBERTURA TOTAL $<10$ & 86 & 81 & 84 \\
\hline & $\%$ COBERTURA <1 & 97 & 89 & 93 \\
\hline & \% COBERTURA 1 A 4 & 93 & 90 & 92 \\
\hline & $\%$ COBERTURA 5 A 9 & 79 & 72 & 76 \\
\hline JOVEN & JOVEN & 70 & 59 & 65 \\
\hline \multirow{3}{*}{$\begin{array}{l}\text { CONTROL } \\
\text { PRENATAL }\end{array}$} & CPN TOTAL & 99 & 101 & 100 \\
\hline & AG ADOLESCENTES $<18$ & 105 & 101 & 103 \\
\hline & $\%$ AG MAYOR 35 AÑOS & 99 & 91 & 95 \\
\hline ADULTOS & ADULTO & 63 & 65 & 64 \\
\hline \multirow{2}{*}{$\mathrm{CCU}$} & CCU 10 A 24 & 47 & 58 & 53 \\
\hline & 25 A 69 & 75 & 73 & 74 \\
\hline SENO & SENO & 57 & 41 & 49 \\
\hline AGUDEZA VISUAL & AGUDEZA VISUAL & 63 & 49 & 56 \\
\hline
\end{tabular}

$P E=$ Protección Específica, $\mathrm{DT}=$ Detección Temprana, $\mathrm{AG}=$ Atención a gestantes, $\mathrm{RN}=$ Recién Nascido, $\mathrm{CCU}=$, $\mathrm{BCG}=, \mathrm{HB}, \mathrm{DPT}, \mathrm{VOP}, \mathrm{HMOPHIL}, \mathrm{TD}$ o TT

A nivel departamental el programa de cobertura óptima, es el programa de detección temprana de alteraciones del embarazo y la segunda mejor cobertura, se observa en el programa de detección temprana de alteraciones del crecimiento y desarrollo en el menor de 10 años, que alcanza el 86\%. Por otro lado la no presencia de muertes maternas en las ZBS, y el incremento del parto institucional del 59,04\% (fuente bases SICAPS) al 93\% (fuente Interventoría USCO 2007), los indicadores de mortalidad infantil en las zonas intervenidas, son significativamente menores a los indicadores departamentales y de la zona rural dispersa, pudiendo concluir que se ha contribuido a mejorar los indicadores.
Como resultado, observamos que para las coberturas de los programas, se promedia $62 \%$ y para los programas prioritarios $73 \%$, para un total de $68 \%$ calificado como DEFICIENTE.

\section{ATENCIÓN APROPIADA Y EFECTIVA}

Durante los procesos de proyección financiera de la Estrategia en el Departamento del Huila, se garantizo el cumplimiento del objeto de las metas propuestas, respondiendo a los principios de eficiencia y equidad. Por tal razón, el indicador se promediara al máximo a expensas de que la investigación no incluya la evaluación financiera de la estrategia. 


\section{RECURSOS HUMANOS APROPIADOS}

Se realizó la evaluación a los coordinadores, con respecto al manejo de los sistemas de información durante el año 2006 que se concluye en $92,7 \%$.

\section{EQUIPO EXTRAMURAL Y EQUIPO BÁSICO DE SALUD (EBS)}

Los EBS, se conformaron de la siguiente manera: 1 Coordinador Municipal, 1 Digitador, 1Medico, 1Enfermero, 1 Auxiliar de Enfermería, 1 Higienista Oral y 3 Agentes Comunitarios (Tabla 5).

Tabla 5. Conformación de Equipos Básicos en Salud

\begin{tabular}{|l|c|c|c|c|}
\hline MUNICIPIO & NUMERO & $\begin{array}{c}\% \\
\mathbf{2 0 0 6}\end{array}$ & $\begin{array}{c}\mathbf{2 0 0 7} \\
\text { NORTE }\end{array}$ & $\begin{array}{c}\% \\
\text { TOTAL }\end{array}$ \\
\hline CENTRO & 17 & 71 & 57 & 66 \\
\hline OCCIDENTE & 11 & 64 & 82 & 73 \\
\hline SUR & 22 & 59 & 68 & 64 \\
\hline TOTAL & 85 & 67 & 67 & 67 \\
\hline
\end{tabular}

La regularidad de la permanencia del personal en la estrategia, fue de aproximadamente un $80 \%$ del tiempo de ejecución.

\section{INTEGRACIÓN CON LAS FESP}

Enla vigilancia epidemiológica de las enfermedades de interés en salud pública, la implementación y operativización de los COVECOM (Comité de Vigilancia Epidemiológica Comunitaria), en las veredas intervenidas, fueron realizados por los EBS, con el reporte de las enfermedades de interés en salud pública y de los indicadores centinela, tomando como referencia los datos suministrados por la ESE o UAE municipal y reportando los casos correspondientes a cada una de las respectivas zonas intervenidas, notificando el 100\% de los casos presentados. Sin embargo, los valores resultantes del indicador, demostraron las falencias que se presentaron en las capacitaciones en vigilancia de eventos de salud pública a la comunidad y la implementación y operativización de los COVECOM. En la mayoría de los casos no obedeció a incumplimiento de los EBS, si no, a las fallas operativas y técnicas del programa, como la poca participación de la comunidad a las actividades de participación comunitaria, a la dificultad de acceso a ciertos territorios, no contar con el servicio de transporte entre otros (Tabla 6).

Tabla 6. Porcentaje de cumplimiento de Integración con las FESP

\begin{tabular}{|l|c|c|c|}
\hline & $\begin{array}{c}\% \\
2006\end{array}$ & $\begin{array}{c}\% \\
\text { 2007 }\end{array}$ & $\begin{array}{c}\% \\
\text { TOTAL }\end{array}$ \\
\hline OCCIDENTE & 94 & 95 & 95 \\
\hline CENTRO & 99 & 57 & 78 \\
\hline NORTE & 59 & 60 & 60 \\
\hline SUR & 78 & 57 & 68 \\
\hline TOTAL & 83 & 67 & 75 \\
\hline
\end{tabular}

\section{INTERSECTORIALIDAD}

Para procurar los mejores resultados, se recomendó amplia socialización de los objetivos, actividades y resultados de la estrategia; reuniones donde se describan las temáticas a tratar y la importancia de la participación, para las soluciones de los problemas de las comunidades intervenidas, socializar el diagnóstico comunitario con la propuesta del plan de acción, socializar los logros, aciertos y beneficios alcanzados para la comunidad en el desarrollo de la estrategia, invitar a las reuniones a los líderes de las comunidades intervenidas (Figura 4).

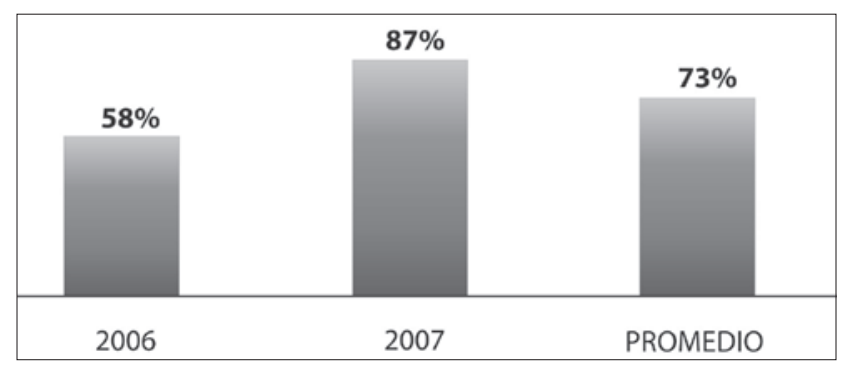

Figura 4. Porcentaje de cumplimiento a las reuniones de concertación

La formulación de los planes de acción veredal, conto con la participación de la comunidad y sus líderes, derivadas del diagnostico comunitario. El plan de acción familiar se concertó con la familia, para modificar los factores de riesgo o para implementar 
las medidas necesarias, para corregir una situación identificada como adversa (Figura 5).

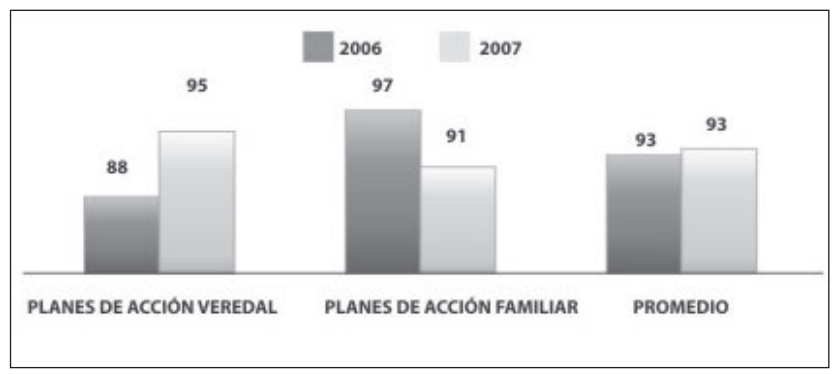

Figura 5. Numero de planes de acción veredal y de acción familiar

El grado en el cual las acciones fueron implementadas, no era obligación contractual de los EBS, su responsabilidadera generarinquietudesenlas reuniones de concertación, para que desde allí se dieran soluciones con el equipo participante tomadores de decisión. Sin embargo, mediante el trabajo intersectorial se logro: identificación de población vulnerable (gestantes y menores de un año) para gestionar afiliación al régimen subsidiado, jornadas de las registradurías para la generación de documentos de identificación, Jornadas de vacunación canina y felina, Inscripción en proyectos municipales de mejoramiento de vivienda, articulación con el programa de Salud Mental, para la intervención de casos identificados de violencia intrafamiliar, articulación con la coordinación del PAB (Plan de Atención Básico),municipal en actividades de participación comunitaria.

\section{MECANISMOS DE PARTICIPACIÓN ACTIVA}

El cumplimiento en el período 2006 fue bajo con respecto al 2007, sin embargo, se presenta un valor promedio de $81 \%$ entre los dos períodos reflejados (Figura 6).

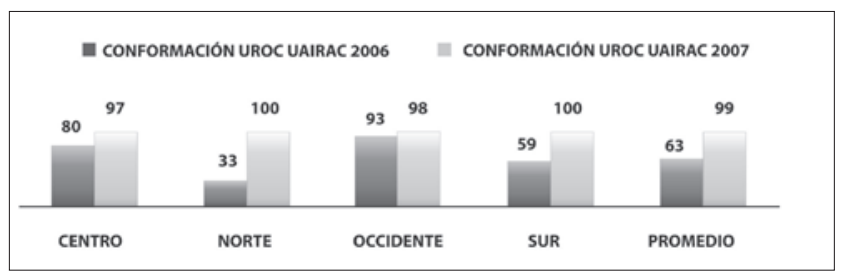

Figura 6. Conformación de UROCS - UAIRAC

Con respecto a la conformación de los Comités de Vigilancia Epidemiológica Comunitaria
(COVECOM), solamente se registran valores correspondientes a la conformación de los comités (Tabla 7 y Figura 7).

Tabla 7. Programas de participación activa

\begin{tabular}{|c|c|c|c|}
\hline PROGRAMA & $\begin{array}{c}\% \\
2006\end{array}$ & $\begin{array}{c}\% \\
2006\end{array}$ & $\begin{array}{c}\% \\
\text { TOTAL }\end{array}$ \\
\hline UROC UAIRAC & 63 & 99 & 81 \\
\hline IAMI AIPEI & 69 & 85 & 77 \\
\hline COVECOM & 54 & 76 & 65 \\
\hline
\end{tabular}

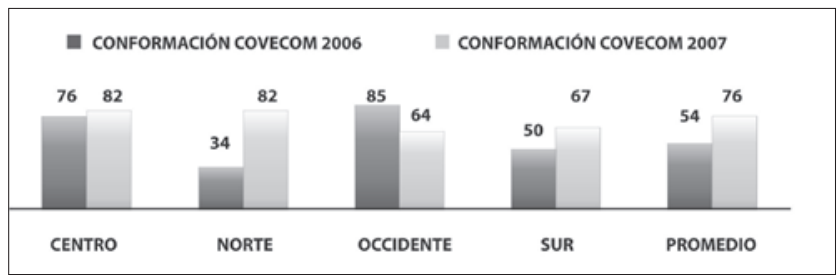

Figura 7. Conformación COVECOM 2006 - 2007

\section{CONCLUSIONES}

La tabla 8 presenta de forma resumida las lecciones aprendidas y recomendaciones de la investigación. Los planificadores deberán involucrar no sólo el sistema de salud, sino también la capacidad de los actores locales y nacionales, para colaborar en el alcance de una visión integrada y crear una infraestructura colaborativa, para desarrollar los sistemas de salud liderados por la APS, que pueden actuar como conductores del sistema de atención a la salud, respondiendo a los problemas comunes de salud, proporcionando un punto focal para la integración de actividades de desarrollo social y económico, y luchando contra la exclusión social, creando vínculos sinérgicos entre los sectores. Las reformas a la salud, implican cambios fundamentales en procesos políticos y de poder, creando además conciencia colectiva, mediante la cual, cada quién se sienta comprometido con la comunidad, con una actitud profundamente solidaria. Todo ello, para la legitimación de las instituciones, la educación, la investigación, la aplicación de conocimientos, el capital físico y el humano, el crédito popular y el apoyo al emprendimiento, serán los pilares de competitividad que aumenten el ingreso y mejoren su distribución, con un conjunto armónico de acciones 
Tabla 8. Lecciones aprendidas y resultados de la investigación.

\begin{tabular}{|c|c|c|c|}
\hline \multirow{2}{*}{$\begin{array}{l}\text { ELEMENTOS } \\
\text { ESENCIALES DE } \\
\text { LA APS }\end{array}$} & \multirow{2}{*}{$\begin{array}{c}\text { FUNCIÓN } \\
\text { ESTRUCTURAL }\end{array}$} & \multicolumn{2}{|c|}{ RESULTADOS } \\
\hline & & LECCIONES APRENDIDAS & RECOMENDACIONES \\
\hline ACCESIBILIDAD & $\begin{array}{l}\text { ELIMINAR } \\
\text { BARRERAS DE } \\
\text { ACCESO }\end{array}$ & $\begin{array}{l}\text { DECISIÓN TRASCENDENTAL } \\
\text { AMBIENTE FAVORABLE PARA IMPULSAR EL } \\
\text { DESARROLLO COMUNITARIO A TRAVÉS DE LA } \\
\text { SALUD; PROCESO SOCIAL OUE ARTICULA Y } \\
\text { FORTALECE LA POLIITICA DEPARTAMENTAL. } \\
\text { SICAPS DETERMINANTES DE LOS MAYORES } \\
\text { FLAGELOS (POBREZA Y VIOLENCIA). } \\
\text { DISMINUCIÓN DEINCIDENCIADELA POBREZA } \\
\text { (- 20\%). } \\
\text { ATENCIÓN DE POBLACIÓN RURAL } 46 \% \\
\text { POLÍTICA DE SEGURIDAD SOCIAL FUE } \\
\text { DETERMINANTE. }\end{array}$ & $\begin{array}{l}\text { REALIZACIÓN DE AJUSTES. } \\
\text { IMPACTAR MÁS EFICIENTEMENTE LA SALUD DE } \\
\text { LAS PERSONAS Y DEL AMBIENTE. } \\
\text { DISEÑO DE POLÍTICA PÚBLICA DEPARTAMENTAL } \\
\text { DE APS }\end{array}$ \\
\hline ACEPTABILIDAD & $\begin{array}{l}\text { SATISFACCIÓN CON LOS } \\
\text { SERVICIOS OFRECIDOS }\end{array}$ & $\begin{array}{l}\text { LAZOS MÁS FUERTES CON LA COMUNIDAD. } \\
\text { FACTORES DETERMINANTES CULTURA, } \\
\text { RELIGIÓN, GRADO DE EDUCACIÓN, } \\
\text { EXPERIENCIAS NEGATIVAS Y } \\
\text { CARACTERÍSTICAS OCUPACIONALES } \\
\text { INTERRUPCIÓN DE LA CONTINUIDAD. }\end{array}$ & $\begin{array}{l}\text { PROPUESTA DINÁMICA Y PERMANENTE. } \\
\text { DISEÑAR LA APLICACIÓN DE LA ENCUESTA Y LA } \\
\text { PUBLICACIÓN DE LOS RESULTADOS OBTENIDOS } \\
\text { PARA } \\
\text { PLANIFICAR LOS PLANES CORRECTIVOS. }\end{array}$ \\
\hline PRIMER CONTACTO & $\begin{array}{l}\text { PUERTA DEENTRADAAL } \\
\text { SISTEMA DE SERVICIOS } \\
\text { SOCIALES Y DE SALUD }\end{array}$ & $\begin{array}{l}\text { PREVISIÓN EN LA ATENCIÓN MEDIANTE UN } \\
\text { PLAN DE ACCIÓN DE VEREDAS Y FAMILIAR. } \\
\text { ALTA ROTACIÓN DE LA COMUNIDAD RURAL. } \\
\text { LAS ESES DEPARTAMENTALES } \\
\text { FORTALECIERON EL SISTEMA DE REFERENCIA } \\
\text { Y CONTRARREFERENCIA，ESPECIALMENTE } \\
\text { EN LA ATENCIÓN A LAS GESTANTES }\end{array}$ & $\begin{array}{l}\text { PARA MEJORAR LA PREVISIÓN EN LA } \\
\text { PROGRAMACIÓN DE LAS ACTIVIDADES. } \\
\text { CADAIPS MUNICIPAL DEBEASUMIREL CONTROL } \\
\text { ESTRICTO. }\end{array}$ \\
\hline $\begin{array}{lr}\text { A T E N C I } & \text { Ó } \\
\text { INTEGRAL } & \text { E } \\
\text { INTEGRADA } & \end{array}$ & $\begin{array}{l}\text { P R O P O R C I O N A } \\
\text { ATENCIÓN INTEGRAL } \\
\text { E INTEGRADA A LA } \\
\text { POBLACIÓN. }\end{array}$ & $\begin{array}{l}\text { ATENCIÓN DE LA COMUNIDAD EN } \\
\text { DONDE ANTERIORMENTE NO LLEGABAN } \\
\text { PROGRAMAS PERMITIENDO COBERTURAS } \\
\text { SATISFACTORIAS. }\end{array}$ & $\begin{array}{l}\text { PARA MAXIMIZAR LA SALUD SE REOUIERE OUE } \\
\text { TODAS LAS PARTES DEL SISTEMA DE SALUD } \\
\text { CONSTRUYAN JUNTOS EN BENEFICIO DE LAS } \\
\text { COMUNIDADES. } \\
\text { CONTRATACIÓN DIRECTA CON LOS NIVELES } \\
\text { LOCALES. }\end{array}$ \\
\hline $\begin{array}{l}\text { ENFOQUE } \\
\text { POBLACIONAL }\end{array}$ & $\begin{array}{lr}\text { EMPLEA LA } & \text { SALUD } \\
\text { PÚBLICA } & \text { PARA } \\
\text { VALORAR LOS RIESGOS } \\
\text { Y PRIORIZAR LAS } \\
\text { INTERVENCIÓN }\end{array}$ & $\begin{array}{l}\text { EL DIAGNÓSTICO ES UN NORTE PARA } \\
\text { EL DESARROLLO DE LAS MISMAS, PUES } \\
\text { IDENTIFICA Y FOCALIZA LAS NECESIDADES } \\
\text { DE INVERSIÓN EN ACCIONES PUNTUALES } \\
\text { DE SEGUIMIENTO Y EVALUACIÓN DE } \\
\text { PROGRAMAS INTEGRALES DE FORMA } \\
\text { AUTOMATIZADA. }\end{array}$ & $\begin{array}{l}\text { VALORAR Y ARTICULAR EL DIAGNÓSTICO DE LA } \\
\text { SITUACIÓN DE LAS COMUNIDADES CON LAS } \\
\text { POLIITICAS REGIONALES. } \\
\text { CONSTRUCCIÓN DE UN DOCUMENTO OUE } \\
\text { CONSOLIDE LA INFORMACIÓN INTEGRAL DEL } \\
\text { DIAGNOSTICO. }\end{array}$ \\
\hline
\end{tabular}




\begin{tabular}{|c|c|c|c|}
\hline \multirow{2}{*}{$\begin{array}{l}\text { ELEMENTOS } \\
\text { ESENCIALES DE } \\
\text { LA APS }\end{array}$} & \multirow{2}{*}{$\begin{array}{c}\text { FUNCIÓN } \\
\text { ESTRUCTURAL }\end{array}$} & \multicolumn{2}{|c|}{ RESULTADOS DE LA INVESTIGACIÓN } \\
\hline & & LECCIONES APRENDIDAS & RECOMENDACIONES \\
\hline $\begin{array}{lr}\text { ÉNFASIS } & \text { EN } \\
\text { PROMOCIÓN } & \text { Y } \\
\text { PREVENCIÓN DE LA } \\
\text { SALUD }\end{array}$ & $\begin{array}{l}\text { PROMOCIÓN DE LA } \\
\text { SALUD Y PREVENCIÓN } \\
\text { DE LAS ENFERMEDAD }\end{array}$ & $\begin{array}{l}\text { ESTAS INTERVENCIONES SON COSTO- } \\
\text { EFECTIVAS, ÉTICAS, EMPODERAN A LAS } \\
\text { COMUNIDADES Y A LOS INDIVIDUOS PARA } \\
\text { EJERCER MAYOR CONTROL SOBRE SU } \\
\text { SALUD. } \\
\text { HAY DEFICIENCIAS EN LA ATENCIÓN DE } \\
\text { LA POBLACIÓN FLOTANTE, PERSONAS } \\
\text { VINCULADAS Y PERSONAS SIN NINGÚN TIPO } \\
\text { DE SEGURIDAD SOCIAL EN SALUD, } \\
\text { LOS DATOS OBTENIDOS NO SUMINISTRANEN } \\
\text { SU TOTALIDAD LA INFORMACIÓN REQUERIDA } \\
\text { Y DIFICULTAN SU EVALUACIÓN }\end{array}$ & $\begin{array}{l}\text { INTEGRACIÓN DE LAS ACCIONES DE PY P QUE SE } \\
\text { DESARROLLAN EN EL PLAN DE SALUD PÚBLICA } \\
\text { YEL POS. } \\
\text { LAS ACCIONES AÚN ESTÁN ENFOCADAS SOBRE } \\
\text { LA ATENCIÓN ESPECIALIZADA Y HOSPITALARIA, } \\
\text { GENERANDO ALTOS COSTOSY } \\
\text { EXACERBAN LAS DESIGUALDADES SOCIALES } \\
\text { EN SALUD. } \\
\text { LOS DOCUMENTOS QUE SOPORTAN LA } \\
\text { ESTRATEGIA REOUIEREN CONCEPTUALIZACIÓN, } \\
\text { SOBRE LA TOTALIDAD LAS BASES TEÓRICAS Y } \\
\text { PRÁCTICAS. }\end{array}$ \\
\hline $\begin{array}{ll}\text { A T E N C I Ó N } \\
\text { APROPIADA } \\
\text { EFECTIVA }\end{array}$ & $\begin{array}{lr}\text { ATENDER } & \text { LAS } \\
\text { NECESIDADES CON } & \text { COITERIODEEFICIENCIA, } \\
\text { CRITERIVIDAD } \\
\text { EFECTIVIDAD } \\
\text { EQUIDAD. }\end{array}$ & $\begin{array}{l}\text { SE GARANTIZÓ QUE LA ATENCIÓN FUERA } \\
\text { EFECTIVA AL LOGRARSE UNA PROYECCIÓN } \\
\text { FINANCIERA DE LOS RECURSOS NECESARIOS. }\end{array}$ & $\begin{array}{l}\text { INVOLUCRAR A SU VEZ A LOS DIFERENTES } \\
\text { ACTORES DEL SGSSS A MEDIANO Y LARGO } \\
\text { PLAZO. } \\
\text { APROVECHAR LA OPORTUNIDAD POLÍTICA DE } \\
\text { CAMBIO QUE DEBE CAPITALIZARSE. }\end{array}$ \\
\hline $\begin{array}{l}\text { REC C U R S O S } \\
\text { H U M A N O S } \\
\text { APROPIADOS }\end{array}$ & EQUIPO DE TRABAJO & $\begin{array}{l}\text { LAAMENAZAMÁSRELEVANTEDELPROGRAMA } \\
\text { SE DIO EN LOS CAMBIOS FRECUENTES EN EL } \\
\text { PERSONAL PROFESIONAL DE LOS EBS. } \\
\text { SE EVIDENCIARON DEFICIENCIAS EN TEMAS } \\
\text { CONCEPTUALES BÁSICOS DE APS } \\
\text { DESCONOCIMIENTO DE LAS POLÍTICAS } \\
\text { PÚBLICAS. } \\
\text { SE ESTABLECIERON MOMENTOS EN DONDE } \\
\text { EL APRESTAMIENTO DE LOS EBS, DEDICO } \\
\text { GRAN PARTE DEL TIEMPO DE EJECUCIÓN DE } \\
\text { LA ESTRATEGIA. } \\
\text { LAS EXPERIENCIAS Y ADQUISICIÓN DE } \\
\text { CONOCIMIENTOS PARA CASI TOO PERSONAS } \\
\text { EN EL MANEJO DE LA ESTRATEGIA } \\
\text { PERMITIRÁ TENER UNA VISIÓN HACIA UNA } \\
\text { ATENCIÓN PREVENTIVA DIFERENTE A LOS } \\
\text { CONCEPTOS. }\end{array}$ & $\begin{array}{l}\text { ESTABILIDAD LABORAL. } \\
\text { ACTUALIZACIÓN EN EL MANEJO INTEGRAL DE } \\
\text { LAS ACTIVIDADES DE GESTANTES. } \\
\text { PROCESO DE INDUCCIÓN Y CAPACITACIÓN DEL } \\
\text { PERSONAL QUE SE VINCULA A LA ESTRATEGIA } \\
\text { Y FIJAR CONDICIONES Y RESPONSABILIDADES A } \\
\text { LA HORA DE SALIR. } \\
\text { ESTABLECER LOS LINEAMIENTOS Y DEFINIR LA } \\
\text { LÍNEA DE AUTORIDAD CON TODO EL PERSONAL } \\
\text { DE LA ESTRATEGIA EN LOS MUNICIPIOS POR } \\
\text { PARTE DE LA ESE REGIONAL, SE RECOMIENDA } \\
\text { DELEGAR LA COORDINACIÓN GENERAL EN UNA } \\
\text { PERSONA CON DISPONIBILIDAD EXCLUSIVA Y } \\
\text { CON AMPLIOS CONOCIMIENTOS EN ATENCIÓN } \\
\text { PRIMARIA EN SALUD. } \\
\text { IMPLEMENTAR UN PROGRAMA ACADÉMICO, } \\
\text { TÉCNICO Y PROFESIONAL EN LA APS. }\end{array}$ \\
\hline $\begin{array}{l}\text { MECANISMOS } \\
\text { DE PARTICIPACIÓN } \\
\text { ACTIVA }\end{array}$ & $\begin{array}{l}\text { INTEGRACIÓN } \\
\text { CON LAS COMUNIDAD }\end{array}$ & $\begin{array}{l}\text { SE PRESENTAN ALGUNAS DIFICULTADES EN } \\
\text { LA RECEPTIVIDAD DE LAS ACTIVIDADES DE } \\
\text { CAPACITACIÓN } \\
\text { FALTA DE FORTALECIMIENTO } \\
\text { INTERINSTITUCIONAL TANTO A NIVEL } \\
\text { CENTRAL COMO MUNICIPAL, QUE APOYE } \\
\text { LAS ORGANIZACIONES COMUNITARIAS } \\
\text { Y DIRECCIONE TODOS LOS PROGRAMAS } \\
\text { SOCIALES DEL ESTADO Y LAS REGIONES }\end{array}$ & $\begin{array}{l}\text { SE DEBE REPLANTEAR LA ESTRUCTURA PARA } \\
\text { EL MANEJO DE ACTIVIDADES DE CAPACITACIÓN } \\
\text { DIRIGIDAS A LAS COMUNIDADES, PARA } \\
\text { LOGRAR EL EFECTO ESPERADO EN LAS MISMAS, } \\
\text { INVOLUCRANDO LA PARTICIPACIÓN ACTIVA, } \\
\text { USO DE ESTÍMULOS }\end{array}$ \\
\hline
\end{tabular}




\begin{tabular}{|c|c|c|c|}
\hline \multirow{2}{*}{$\begin{array}{c}\text { ELEMENTOS } \\
\text { ESENCIALES } \\
\text { DE LA APS }\end{array}$} & \multirow{2}{*}{$\begin{array}{c}\text { FUNCIÓN } \\
\text { ESTRUCTURAL }\end{array}$} & \multicolumn{2}{|c|}{ RESULTADOS DE LA INVESTIGACIÓN } \\
\hline & & LECCIONES APRENDIDAS & RECOMENDACIONES \\
\hline $\begin{array}{l}\text { INTEGRACIÓN } \\
\text { CON LAS FESP }\end{array}$ & $\begin{array}{l}\text { V I G I L A N C I A } \\
\text { EPIDEMIOLÓGICA PARA } \\
\text { LAS INVESTIGACIÓN } \\
\text { Y } \quad \text { EVALUACIÓN, } \\
\text { GARANTÍA DE CALIDAD } \\
\text { PARA EL DESARROLLO } \\
\text { INSTITUCIONAL A LO } \\
\text { LARGO DEL SISTEMA DE } \\
\text { SALUD. }\end{array}$ & $\begin{array}{l}\text { SE OBSERVAN DIFICULTADES EN LOS } \\
\text { TIEMPOS CORTOS DE INTERVENCIÓN } \\
\text { OUE DIFICULTAN LA REALIZACIÓN DEL } \\
\text { SEGUIMIENTO ADECUADO. } \\
\text { BAJO GRADO DE ACOPLAMIENTO ENTRE } \\
\text { LAS ENTIDADES DE SALUD ESPECIALMENTE } \\
\text { LAS DE PRIMER NIVEL Y LOS EOUIPOS DE LA } \\
\text { ESTRATEGIA. } \\
\text { NO EXISTIÓ UN ENLACE ENTRE LOS } \\
\text { PROGRAMAS DE SALUD LIDERADOS POR } \\
\text { SSD Y LA ESTRATEGIA }\end{array}$ & $\begin{array}{l}\text { MONITOREO DE LA EVALUACIÓN Y SEGUIMIENTO } \\
\text { CONSTANTE. } \\
\text { GENERAR MESAS DE TRABAJO ENTRE LOS } \\
\text { RESPONSABLES DE LA ESTRATEGIA Y CADA } \\
\text { UNO DE LOS COORDINADORES DE LAS ÁREAS } \\
\text { DE SALUD PÚBLICA. } \\
\text { PARTICIPACIÓN Y RETROALIMENTACIÓN } \\
\text { CON LAS UNIDADES DE ANÁLISIS LOCALES Y } \\
\text { DEPARTAMENTALES. }\end{array}$ \\
\hline $\begin{array}{l}\text { ACCIONES } \\
\text { INTERSECTORIALES }\end{array}$ & 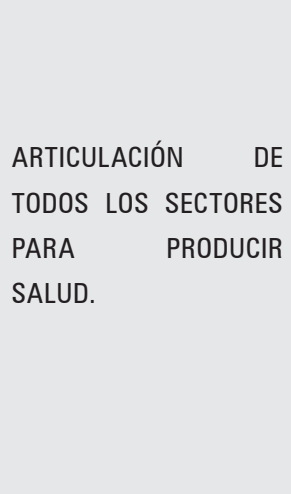 & $\begin{array}{l}\text { CON EL NUEVO ENFOQUE DE LA ATENCIÓN } \\
\text { PRIMARIA EN SALUD RENOVADA, POCA } \\
\text { PARTICIPACIÓN YLAFALTA DE COMPROMISO } \\
\text { POR PARTE DE LOS DIFERENTES ENTES } \\
\text { INSTITUCIONALES Y NO INSTITUCIONALES, } \\
\text { DESCONOCIENDO EL PROPÓSITO MISMO DE } \\
\text { LA PROPUESTA. }\end{array}$ & $\begin{array}{l}\text { EL ESOUEMA DE ORGANIZACIÓN Y } \\
\text { ARTICULACIÓN DE LA ESTRATEGIA DEBERÍA } \\
\text { DIRECCIONARSE DESDE UNA GERENCIA DE LA } \\
\text { POLIITICA SOCIAL QUEGARANTICEEL CONCEPTO } \\
\text { DE LO HUMANO BASADA EN LOS VALORES } \\
\text { DE EOUIDAD Y SOLIDARIDAD, REGIDO POR UN } \\
\text { CONCEJO DE POLIITICA SOCIAL EL CUAL BRINDA } \\
\text { LOS LINEAMIENTOS PARA LA PARTICIPACIÓN Y } \\
\text { EL CONTROL SOCIAL EN LA RESPONSABILIDAD } \\
\text { COMPARTIDA DE LA COMUNIDAD Y LAS } \\
\text { INSTITUCIONES. }\end{array}$ \\
\hline
\end{tabular}

sociales que deben producir impactos positivos en condiciones de vida.

La estrategia ha dado pasos fundamentales en el diagnóstico como parte del proceso, pero aún no se ha logrado la labor de integrar acciones, que permitan dar solución y no se cuenta con los mecanismos para intervenir dicha problemática.

\section{AGRADECIMIENTOS}

El autor expresa sus agradecimientos al Doctor José Domingo Alarcón por el apoyo para la realización y culminación de este proyecto y a la Universidad Surcolombiana por el respaldo académico de esta investigación.

\section{REFERENCIAS BIBLIOGRÁFICAS}

1. Macinko J, Montenegro H, Nebot Adell C, Etienne C y Grupo de Trabajo de Atención Primaria de Salud de la Organización Panamericana de la
Salud. La renovación de la atención primaria de salud en las Américas. Revista Panamericana Salud Publica. 2007; 21(2/3):73-84.

2. Organización Panamericana de la Salud. Atención Primaria de Salud en las Américas. Enseñanzas extraídas a lo largo de 25 años y los retos futuros. Washington. PAHO 2003.

3. Casas JA. La experiencia de SPP en la región de las Américas: Implantación de los programas. Renovación de salud para todos. Washington, DC: PAHO, 1996.

4. Primer Modelo Regional de Atención Primaria en Salud con Enfoque Familiar. Salud puerta a Puerta Secretaria de Salud Departamental del Huila. Secretaria de Salud Departamental del Huila USCO. Documento. Neiva, 2006.

5. Hospital Departamental Hernando Moncaleano Perdomo, Huila Unido, Secretaria de Salud Departamental. Salud Puerta a Puerta APS en el Huila Una experiencia de APS en el departamento del Huila. Neiva Huila 2007. 